01

\title{
Влияние электрического и магнитного полей на угловое распределение интенсивности света, рассеянного холодным атомным ансамблем
}

\author{
(C) Н.В. Ларионов, И.М. Соколов \\ Санкт-Петербургский политехнический университет Петра Великого, \\ 195251 Санкт-Петербург, Россия \\ e-mail: larionov.nickolay@gmail.com
}

Поступила в редакцию 19.03.2019 г.

В окончательной редакции 19.03.2019 г.

Принята к публикации 16.05.2019 г.

\begin{abstract}
Теоретически исследовано угловое распределение интенсивности света, рассеянного оптически плотным холодным ансамблем, внесенным в однородное постоянное электрическое или магнитное поле. Проанализированы парциальные вклады рассеяния различной кратности в полную интенсивность отдельных поляризационных компонент вторичного излучения. Показано, что внешние статические поля, вызывая анизотропию среды, приводят к изменению как углового распределения этого излучения, так и относительного вклада рассеяния различных порядков.
\end{abstract}

Ключевые слова: холодный атомный ансамбль, некогерентное многократное рассеяние, оптическая анизотропия.

DOI: $10.21883 /$ OS.2019.09.48191.116-19

\section{Введение}

Прогресс в области лазерного охлаждения атомных ансамблей сделал их в настоящее время одним из важных инструментов при исследовании фундаментальных физических процессов в атомной физике и квантовой оптике. Такие ансамбли рассматриваются также как весьма перспективные для широкого круга практических применений в метрологии и квантовой информатике [1-6]. Активное использование атомных ансамблей, охлажденных до низких и сверхнизких температур, делает актуальной задачу управления их свойствами, в первую очередь оптическими. Одним из наиболее простых и в то же время весьма эффективных методов является применение управляющих статических электрического или магнитного полей. Помимо управления свойствами ансамбля важной задачей является диагностика его состояния. Такая диагностика в большинстве случаев проводится оптическими методами на основе анализа характера рассеяния ансамблем электромагнитного излучения.

Интерпретация результатов экспериментов по рассеянию света холодными атомными облаками усложнена тем обстоятельством, что низкие температуры приводят к малой ширине линии атомного перехода и большим сечениям одноатомного рассеяния. Поэтому даже при сравнительно малых размерах атомные ансамбли имеют большую оптическую толщину, и мы имеем дело с многократным рассеянием света внутри такого ансамбля. Кроме этого, рассматриваемые ансамбли являются пространственно неоднородными. Задача еще более усложняется при необходимости учета оптической анизотропии, вызванной внешним стационарным управляющим полем.

Влияние последней на спектральные и поляризационные особенности рассеяния в описываемых условиях было подробно исследовано в работах [7-9]. При этом основное внимание в этих работах было сосредоточено на роли интерференционных эффектов, проявляющихся в таких эффектах, как когерентное обратное рассеяние (КОР) [10-14]. Этот эффект состоит в наличии резко анизотропной добавки к сечению рассеяния, которая отлична от нуля в узком конусе вблизи направления, противоположного направлению падающей на среду волны. Явление КОР чрезвычайно интересно с физической точки зрения и может по-разному проявляться в зависимости от конкретных условий [15-19], но практически никогда не используется для целей диагностики. Это связано со сложностью его экспериментального наблюдения. В подавляющем большинстве реальных экспериментов измеряются характеристики света, рассеянного вне конуса КОР (как, впрочем, и вне конуса когерентного релеевского рассеяния вперед), под некоторым углом к направлению распространения пробного излучения [19-23].

Основная цель настоящей работы - теоретически исследовать влияние управляющих статических полей на характер некогерентного многократного рассеяния света ансамблем холодных атомов. Мы проанализируем изменение поляризационных свойств при таком рассеянии, а также угловое распределение вторичного излучения во всем диапазоне углов. Для более ясного понимания механизма формирования этого излучения 
помимо его полной интенсивности будут рассчитаны парциальные вклады рассеяния различной кратности.

\section{Теоретический подход к описанию рассеяния света}

Описание взаимодействия электромагнитного излучения с атомами будем проводить, предполагая выполненными условия, имеющие место в типичных экспериментах с атомными ансамблями, приготовленными в магнитооптических ловушках. Концентрация атомов $n$ в таких ансамблях является настолько малой, что можно не учитывать эффекты ближнего поля и процессы рекуррентного рассеяния [24-28]. Температура облака составляет величину порядка несколько десятков микрокельвин. При этом типичная величина допплеровского уширения в десятки раз меньше естественной ширины возбужденных состояний. Это позволяет воспользоваться моделью неподвижных атомов. Интенсивность пробного излучения, падающего на атомный ансамбль, в подавляющем большинстве экспериментов выбирается малой, чтобы избежать эффектов насыщения и разогрева облака. В таких условиях всеми нелинейными оптическими явлениями можно пренебречь. Кроме этого, как отмечалось выше, обычно измеряются характеристики света, некогерентно рассеянного вне конуса релеевского рассеяния вперед. Таким образом, далее в этой работе нас не будет интересовать когерентная составляющая рассеянного света, определяемая средним значением напряженности поля в рассеянной волне. В расчетах мы ограничимся вычислением интенсивности различных поляризационных компонент света, испытавшего некогерентное рассеяние на атомах рассматриваемого ансамбля.

Сделанные приближения позволяют использовать при расчете методы диаграммной техники КонстантиноваПереля-Келдыша для неравновесных систем [29,30]. Подробное описание используемого подхода было дано в нескольких наших работах $[8,31]$, и здесь отметим лишь основные его особенности.

Диаграммный метод дает возможность представить интенсивность поляризационных компонент излучения в виде суммы вкладов, определяемых рассеянием различной кратности. Эволюция излучения в среде при этом может рассматриваться как цепочка из последовательных актов рассеяния света на невзаимодействующих между собой атомах. Часть актов рассеяния происходит без изменения моды излучения и связана с релеевским рассеянием вперед. Часть сопровождается изменением направления распространения излучения и его поляризации. Эти процессы принято называть некогерентными. Распространение света между актами некогерентного рассеяния описывается введением запаздывающей функции Грина, учитывающей процессы когерентного рассеяния вперед внутри атомной среды и, таким образом, учитывающей ослабление излучения, изменение его фазы и, возможно, поляризации без изменения направления распространения. Заметим, что используемая нами диаграммная техника может служить формальным обоснованием подхода, получившего название метода случайных блужданий $[22,32,33]$ и основанного на моделирование переноса излучения методами Монте-Карло.

Применение диаграммного метода с учетом сделанных приближений позволяет выразить вклад $N$-кратного некогерентного рассеяния через $3 N$-кратный интеграл по координатам всех атомов. Ключевыми элементами, определяющими соответствующий вклад, как следует из вышеизложенного, являются амплитуда (матрица) однократного рассеяния и запаздывающая функция Грина. Явный вид этих величин приведем для рассматриваемого в работе случая атомов, возбуждаемых на переходе $J_{g}=0->J_{e}=1$.

Матрица рассеяния, записанная в циклическом базисе, имеет следующий вид:

$$
\alpha_{i}^{j}(\omega)=\frac{\left\|d_{e g}\right\|}{3 \hbar} \sum_{\nu=0, \pm 1} \frac{\left(\mathbf{u}^{i} \cdot \mathbf{e}_{\nu}\right)\left(\mathbf{e}^{\nu} \cdot \mathbf{u}_{j}\right)}{\left(\omega-\omega_{\nu}\right)+i \gamma / 2},
$$

где $\left\|d_{e g}\right\| \equiv\left\|d_{J_{g} J_{e}}\right\|-$ приведенный дипольный матричный элемент, не зависящий от магнитных квантовых чисел $M_{e}, M_{g}$ двух рассматриваемых подуровней; $\mathbf{e}_{v}$ и $\mathbf{e}^{v}$ - ко- и контравариантные компоненты циклических ортов. Единичные векторы $\mathbf{u}_{j}$ и $\mathbf{u}^{i}=\mathbf{u}_{i}^{*}$ определяют поляризацию падающего и рассеянного фотонов соответственно, $\gamma$ - ширина линии возбужденного состояния атома, $\omega-$ частота рассеиваемого фотона, $\omega_{v}$ частота атомного перехода

$$
\begin{aligned}
\left|J_{g}=0, M_{g}=0\right\rangle & \equiv\left|M_{g}=0\right\rangle->\left|J_{e}=1, M_{e}=0, \pm 1\right\rangle \\
& \equiv\left|M_{e}=0, \pm 1\right\rangle
\end{aligned}
$$

с учетом зеемановского или штарковского сдвигов, обусловленных влиянием внешних статических полей. При этом индекс $v$ может принимать три значения $v=M_{e}-M_{g}=0, \pm 1$ в зависимости от магнитных квантовых чисел состояний, между которыми происходит переход.

Функция Грина, описывающая распространение света в произвольном направлении по отношению к вектору напряженности внешнего постоянного поля, может быть выражена через тензор диэлектрической восприимчивости $\tilde{\chi}_{i}^{j}(\mathbf{r}, \omega) \equiv \tilde{\chi}_{i}^{j}(\mathbf{r}, \omega ; \alpha, \beta, \gamma)$, определенный в системе координат с осью $z^{\prime}$, задаваемой этим направлением (знак тильда над $\chi$, см. [8]). Здесь $\alpha, \beta, \gamma-$ углы Эйлера [34], определяющие поворот штрихованной системы координат $\left(x^{\prime}, y^{\prime}, z^{\prime}\right)$ относительно лабораторной системы координат $(x, y, z)$, с осью $z$ вдоль постоянного поля. При $z^{\prime}=z$ этот тензор имеет наиболее простой вид

$$
\chi_{i}^{j}(\mathbf{r}, \omega)=-\delta_{i}^{j} \frac{\left\|d_{g e}\right\|^{2}}{3 \hbar} \frac{n(\mathbf{r})}{\left(\omega-\omega_{i}\right)+i \gamma / 2} .
$$


В случае постоянного и однородного внешнего поля направление главных осей тензора $\tilde{\chi}_{i}^{j}(\mathbf{r}, \omega)$ одинаково в различных точках атомного облака, а зависимость $\tilde{\chi}_{i}^{j}(\mathbf{r}, \omega)$ от $\mathbf{r}$ обусловлена только пространственной неоднородностью ансамбля. В этом случае выражение для медленно меняющейся амплитуды запаздывающей функции Грина может быть найдено в аналитическом виде и записано через фазовые интегралы вдоль направления распространения излучения:

$$
\begin{aligned}
& X_{i}^{j}\left(\mathbf{r}_{1}, \mathbf{r}_{2} ; \omega\right)=\left\{\exp \left[2 \pi i k \int_{\mathbf{r}_{2}}^{\mathbf{r}_{1}} \tilde{\tilde{\chi}}(\mathbf{r}, \omega) d s\right]\right\}_{i}^{j}, \\
& \tilde{\tilde{\chi}}(\ldots)= \\
& \left(\begin{array}{ll}
\tilde{\chi}_{+1}^{+1}(\ldots ; \alpha, \beta, \gamma) & \Sigma \tilde{\chi}(\ldots ; \alpha, \beta, \gamma) \\
\Sigma \tilde{\chi}^{*}(\ldots ; \alpha, \beta, \gamma) & \tilde{\chi}_{-1}^{-1}(\mathbf{r}, \omega ; \alpha, \beta, \gamma)
\end{array}\right)
\end{aligned}
$$

где

$$
\begin{aligned}
\Sigma \tilde{\chi}(\ldots ; \alpha, \beta, \gamma)= & \exp (i \gamma) \tilde{\chi}_{+1}^{-1}(\ldots ; \alpha, \beta, \gamma) \\
& +\sum_{i= \pm 1} \tilde{\chi}_{i}^{i}(\ldots ; \alpha, \beta, \gamma) / 2
\end{aligned}
$$

ко- и контравариантные индексы $i, j$ относятся к матрице, получаемой после разложения экспоненты по формуле, аналогичной формуле Эйлера, и справедливой для матриц размерности $2 \times 2$ :

$$
\{\exp [i a(\mathbf{n} \hat{\boldsymbol{\sigma}})]\}_{i}^{j}=\cos (a) \delta_{i}^{j}+i \sin (a)(\mathbf{n} \hat{\boldsymbol{\sigma}})_{i}^{j} .
$$

Здесь $\hat{\boldsymbol{\sigma}}=\left(\hat{\sigma}_{x}, \hat{\sigma}_{y}, \hat{\sigma}_{z}\right), \hat{\sigma}_{i}-$ матрицы Паули и $\mathbf{n}-$ вектор единичной длины. В системе координат с осью $z^{\prime} \|\left(\mathbf{r}_{1}-\mathbf{r}_{2}\right)$ в направлении распространения луча света циклические индексы принимают только два значения $i, j= \pm 1$.

Знание явных аналитических выражений для функции Грина и матрицы рассеяния позволяет рассчитать вклад произвольной цепочки атомов в интенсивность рассеянного света. Вклад рассеяния $N$-й кратности дается усреднением по всем возможным цепочкам, содержащим $N$ атомов. Это усреднение эквивалентно вычислению определенного интеграла, которое в настоящей работе выполнялось с помощью метода статистических испытаний Монте-Карло с использованием метода существенной выборки [35].

\section{Результаты расчетов}

Конкретные расчеты проведены для атомного ансамбля, атомы которого имеют случайное сферически симметричное гауссово пространственное распределение с радиусом $r_{0}=50 \lambda$. Оптическая толщина, равная $b=\sqrt{2 \pi} n_{0} \sigma_{0} r_{0}$, где $\sigma_{0}=6 \pi \lambda^{2}-$ резонансное сечение рассеяния на одном атоме, выбиралась равной 5.
Здесь $\lambda=\lambda / 2 \pi-$ длина волны пробного излучения, деленная на $2 \pi$. В качестве примера ниже рассмотрим две из всех возможных схем поляризационного анализа. Первая соответствует регистрации излучения с той же спиральностью $H$, что и у падающего фотона (схема $H \| H)$. Вторая рассматриваемая схема предполагает анализ рассеянного света с поляризационной компонентой, ортогональной исходной $(H \perp H)$. Величины статических полей будем задавать величиной вызванного ими расщепления мультиплета возбужденного состояния. Соответствующие величины для электрического $\Delta_{E}$ и магнитного $\Delta_{H}$ полей будем нормировать на ширину линии $\gamma$. Конкретные расчеты проведем для достаточно сильных полей $\Delta_{E}=10 \gamma$ и $\Delta_{H}=10 \gamma$, когда их влияние проявляется в максимальной степени.

Пробное излучение во всех обсуждаемых расчетах предполагается направленным вдоль постоянного поля и считается резонансным соответствующему переходу. Конкретные расчеты проведены для левополяризованного света, так что этим переходом является переход

$$
\left|M_{g}=0\right\rangle->\left|M_{e}=-1\right\rangle .
$$

На рис. 1, $а$ для двух рассматриваемых поляризационных схем представлено угловое распределение полной интенсивности рассеянного света при включенных и выключенных статических полях. Из графиков видно, что включение полей увеличивает интенсивность рассеянного света в широком диапазоне углов. Однако в случае включенного магнитного поля для углов рассеяния вблизи направления назад в схеме $H \| H$ и направления вперед в схеме $H \perp H$ имеет место обратная ситуация интенсивность уменьшается и практически полностью исчезает для рассеяния строго назад и строго вперед для соответствующих поляризационных схем. Это объясняется полным отсутствием заселения тех возбужденных зеемановских атомных подуровней, переход с которых в основное состояние дает вклад в эти поляризационные компоненты.

Заметим также, что учет так называемых перекрестных диаграмм, проведенный в нашем расчете [8,31], позволил учесть влияние интерференции при многократном рассеянии и, таким образом, воспроизвести конус обратного рассеяния.

Такое влияние управляющих полей на интенсивность различных поляризационных компонент рассеянного света можно объяснить, проанализировав угловые распределения для парциальных вкладов рассеяния различной кратности. На рис. $1, b$ и рис. 2, $a, b$ при тех же параметрах облака, что и на рис. 1, $a$, представлены угловые распределения для первого и второго, шестого порядков рассеяния соответственно. Из графиков видно, что в случае магнитного поля для схемы $H \| H(H \perp H)$ и углов рассеяния вблизи направления назад (вперед) интенсивность света при увеличении порядка рассеяния остается такой же пренебрежимо малой, как и в случае вклада однократного рассеяния. Это связано с 

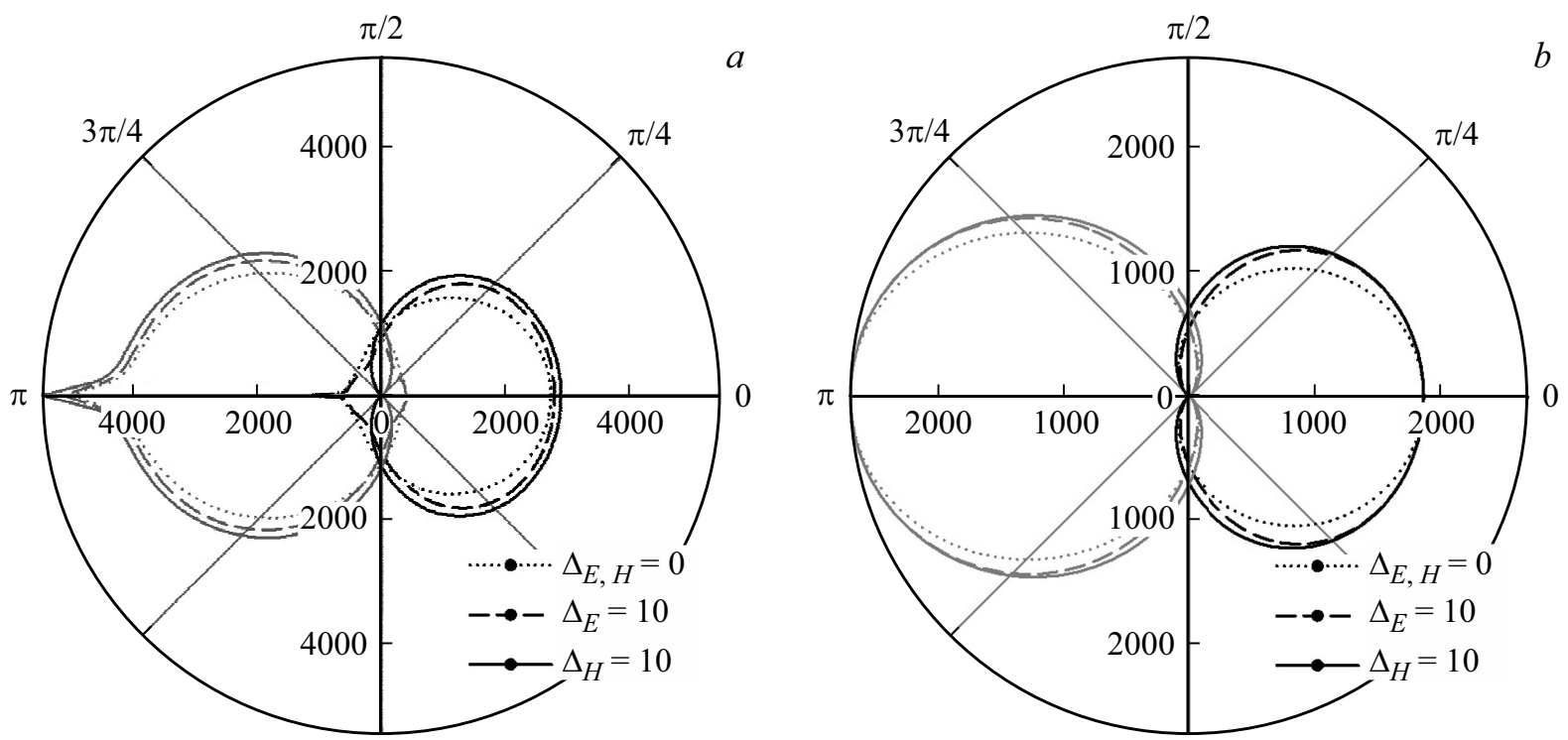

Рис. 1. (a) Угловое распределение полной интенсивности рассеянного света (относительные единицы). (b) Парциальный вклад однократного рассеяния. $H \| H-$ черные кривые, $H \perp H-$ серые кривые.
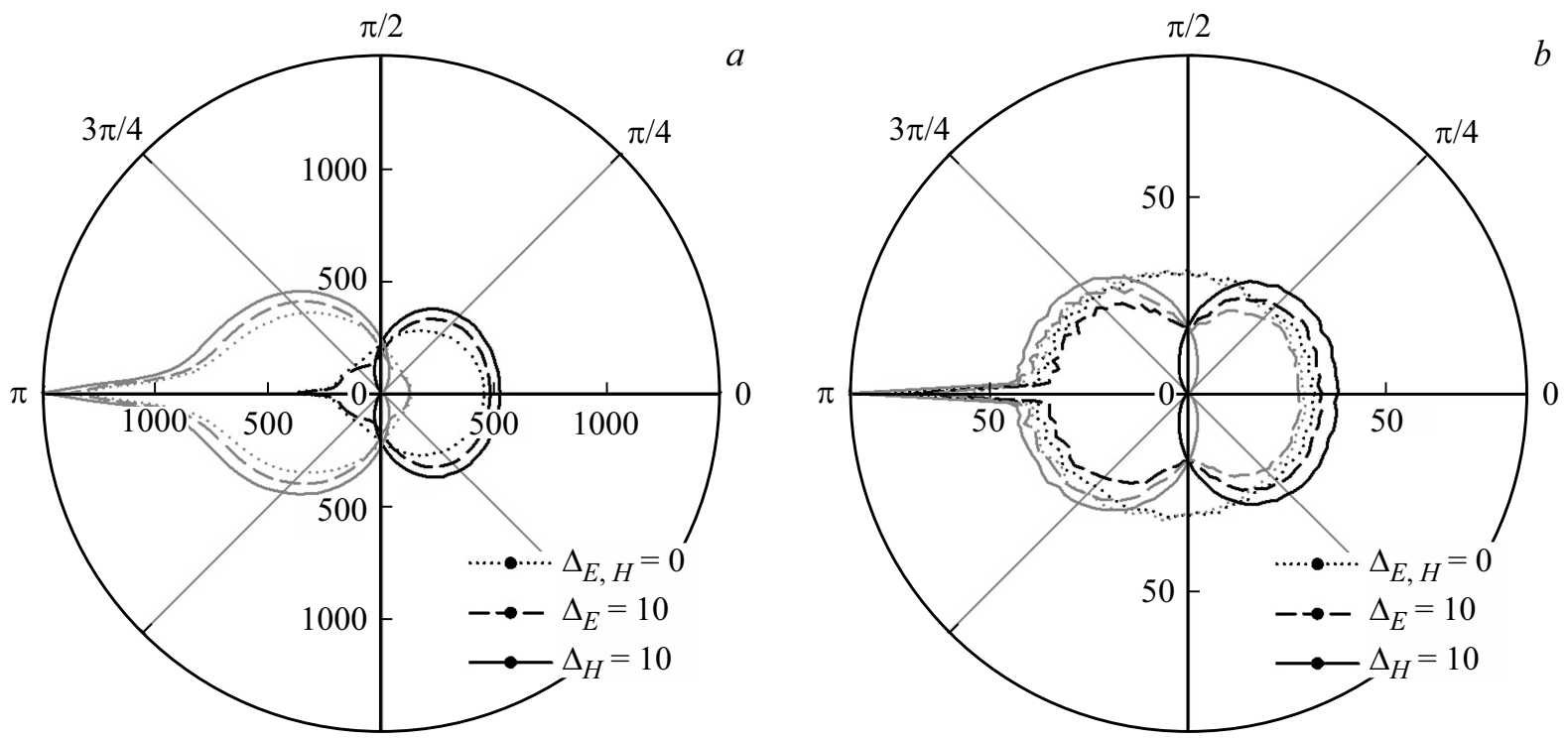

Рис. 2. Угловые распределения интенсивности $(a)$ двукратного, $(b)$ шестикратного рассеяний света. $H \| H-$ черные кривые, $H \perp H-$ серые кривые.

тем, что сильное $\Delta_{H} \gg 1$ зеемановское расщепление уровней обусловливает исчезновение каналов рассеяния, позволяющих вторичному фотону рассеяться на атоме в направлении назад (вперед).

В случае электрического поля в силу только частичного снятия вырождения возбужденного состояния атома вторичный фотон может рассеяться на двух переходах: $\left|M_{g}=0\right\rangle->\left|M_{e}= \pm 1\right\rangle$. Для поляризационной схемы $H \| H$ это обусловливает существенный по сравнению с вкладом однократного рассеяния вклад второго и более высоких порядков рассеяния в полную интенсивность света, рассеянного в направлении, близком к направлению назад.
Для рассеяния вбок вблизи угла $\theta=3 \pi / 4$ для поляризационной схемы $H \perp H$ угловое распределение полной интенсивности света качественно повторяет угловое распределение однократного рассеяния. Однако видно, что статические поля для данного направления увеличивают полную интенсивность рассеянного света (рис. 3,c). В случае другой поляризационной схемы $H \| H$, как это следует из сравнения рис. $1, b$ с рис. 2 , угловая зависимость полной интенсивности света для случаев $\Delta_{E, H}=0, \Delta_{E} \neq 0$ в основном сформирована порядками рассеяния, начиная со второго, а для случае $\Delta_{H} \neq 0$ основной вклад обусловлен однократным рассеянием. 

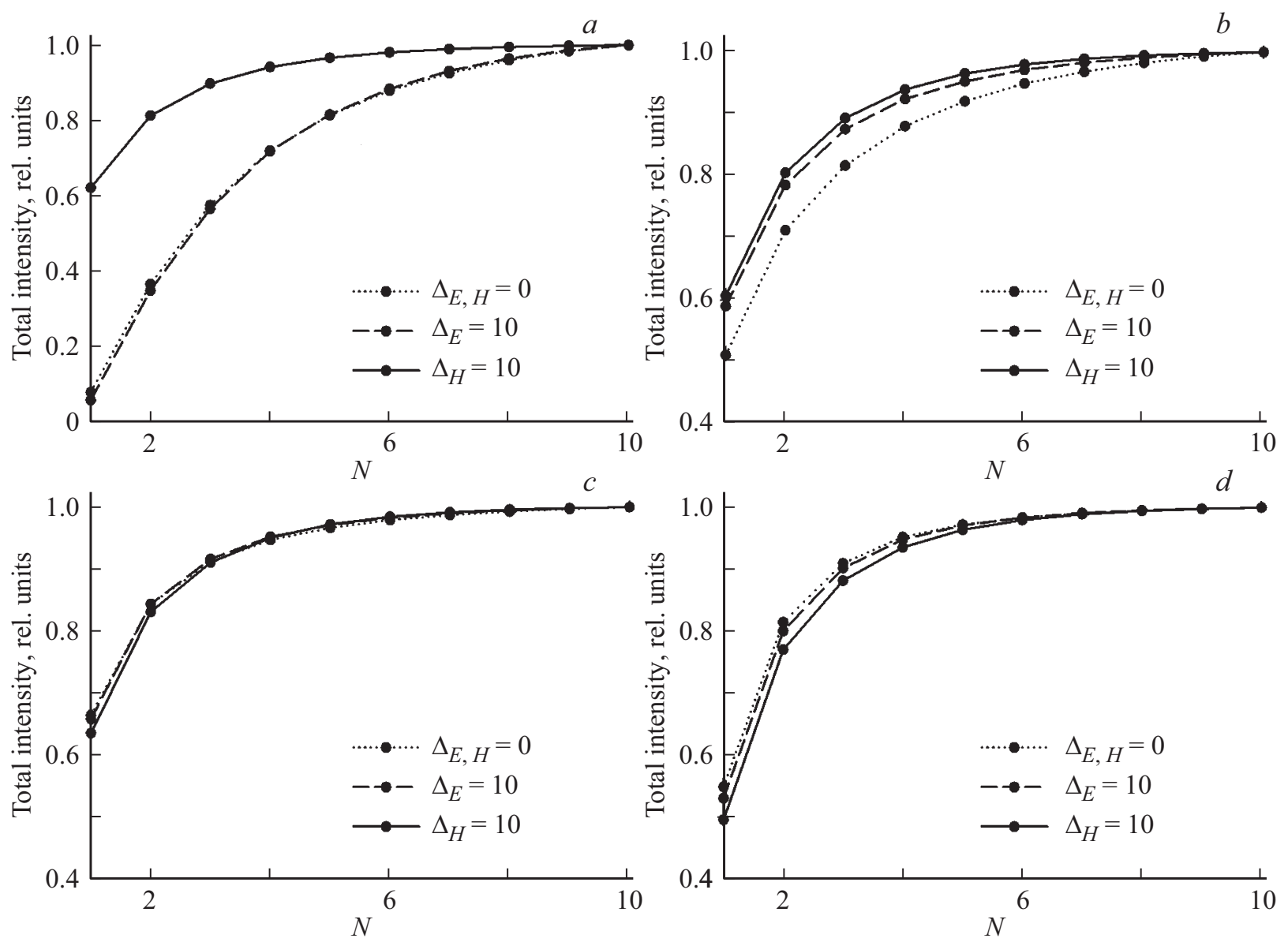

Рис. 3. Зависимость нормированной полной интенсивности рассеянного света от числа $N$ учтенных в расчете актов некогерентного рассеяния, $H \| H . \theta=\pi / 4(a), \theta=\pi / 2(b), \theta=3 \pi / 4(c), \theta=\pi(d)$.

Влияние управляющих полей на интенсивности различных поляризационных компонент света, рассеянного на углы, близкие к $\theta=\pi / 4$, характеризуется аналогичными закономерностями. При рассеянии на углы, близкие к $\theta=\pi / 2$, интенсивности обеих поляризационных компонент совпадают в силу симметрии схемы. Причем эти интенсивности увеличиваются при включении магнитного поля и уменьшаются при включении электрического поля.

На рис. 3 для четырех углов рассеяния $\theta=\pi / 4, \pi / 2$, $3 \pi / 4, \pi$ и поляризационной схемы $H \perp H$ иллюстрируется зависимость нормированной полной интенсивности рассеянного света от числа $N$ учтенных в расчете актов некогерентного рассеяния. Графики дают представление об относительной роли различных порядков рассеяния в формировании полной интенсивности рассеянного в данном направлении света. Эти графики подтверждают выводы о поведении углового распределения полной интенсивности света, сделанные выше на основе анализа угловых распределений для отдельных порядков рассеяния. Так, из рис. $3, a$ видно, что при рассеянии на угол $\theta=\pi / 4$ основной вклад в полную интенсивность в случае $\Delta_{H} \neq 0$ вносят несколько первых порядков рассеяния (рис. $1, b$, рис. $2, a, b$ и пояснения к ним). А для случаев $\Delta_{E, H}=0, \Delta_{E} \neq 0$ роль однократного рассеяния в формировании полной интенсивности мала. При этом магнитное поле существенно усиливает относительный вклад малых порядков рассеяния, а электрическое поле влияет слабо, несколько его ослабляя. Для рассеяния вбок на угол $\theta=\pi / 2$ (рис. $3, b$ ) внешние статические поля усиливают относительный вклад практически всех порядков рассеяния, существенных для рассматриваемых параметров облака. В случае магнитного поля это усиление немного больше. На рис. 3, $d$ представлены кривые для рассеяния строго назад. Особенности этого случая связаны с формированием интерференционной составляющей рассеянного света, ответственного за КОР, и подробно обсуждались в работе [8] для несколько иной геометрии атомного ансамбля.

\section{Заключение}

Рассмотрено влияние внешних электрического и магнитного полей на угловое распределение света, рассеянного атомным ансамблем, охлажденным до субдопплеровских температур в магнитооптической ловушке. Показано, что внешние статические поля за счет внесения 
анизотропии, обусловленной полным или частичным снятием вырождения возбужденного энергетического уровня атома, могут существенно модифицировать угловое распределение рассеянного света. При этом они изменяют соотношение вкладов рассеяния различной кратности, и это изменение различно для разных направлений вторичного излучения.

В заключение отметим, что еще одним эффективным способом управления оптическими свойствами может стать активно обсуждаемое в последнее время использование квазирезонансных управляющих полей, приводящих к таким эффектам как электромагнитно индуцированная прозрачность [2,3,36-38] или когерентное пленение населенностей $[39,40]$.

\section{Финансирование работы}

Работа выполнена при финансовой поддержке Российского научного фонда, проект № 17-12-01085.

\section{Благодарности}

Результаты работы были получены с использованием вычислительных ресурсов суперкомпьютерного центра Санкт-Петербургского политехнического университета Петра Великого (http://www.spbstu.ru).

\section{Конфликт интересов}

Авторы заявляют, что у них нет конфликта интересов.

\section{Список литературы}

[1] Marangos J.P. // J. Mod. Opt. 1998. V. 45. P. 471.

[2] Lukin M.D. // Rev. Mod. Phys. 2003. V. 75. P. 457.

[3] Fleischhauer M., Imamoglu A., Marangos J.P. // Rev. Mod. Phys. 2005. V. 77. P. 633.

[4] Fallani L., Kastberg A. // Europhys. Lett. 2015. V. 110. P. 53001.

[5] Ketterle W. // Nature Phys. 2015. V. 11. P. 982.

[6] Chang D.E., Douglas J.S., Gonzáles-Tudela A., Hung C.-L., Kimble H.J. // Rev. Mod. Phys. 2018. V. 90. P. 031002.

[7] Соколов И.М. // ЖЭТФ. 2017. Т. 152. В. 3. С. 453.

[8] Ларионов Н.В., Соколов И.М. // ЖЭТФ. 2018. Т. 154. В. 2. C. 310.

[9] Ларионов Н.В., Соколов И.М., Фофанов Я.А. // Известия РАН. Серия Физическая. 2019. Т. 83. № 3. С. 302.

[10] Кузьмин В.Л., Романов В.П. // УФН. 1996. Т. 166. В. 3. C. 247.

[11] Sheng $P$. Introduction to Wave Scattering, Localization and Mesoscopic Phenomena. San Diego: Academic Press, 1995. $339 \mathrm{p}$.

[12] Labeyrie G. // Mod. Phys. Lett. B. 2008. V. 22. P. 73.

[13] Aegerter C.M., Maret G. // Prog. Opt. 2009. V. 52. P. 1.

[14] Kupriyanov D.V., Sokolov I.M., Havey M.D. // Phys. Rep. 2017. V. 671. P. 1-60.

[15] Kupriyanov D.V., Sokolov I.M., Havey M.D. // Opt. Commun. 2004. V. 243. P. 165.
[16] Shatokhin V., Muller C.A., Buchleitner A. // Phys. Rev. Lett. 2005. V. 94. P. 043603.

[17] Gremaud B., Wellens T., Delande D., Miniatura C. // Phys. Rev. A. 2006. V. 74. P. 033808.

[18] Chaneliere T., Wilkowski D., Bidel Y., Kaiser R., Miniatura C. // Phys. Rev. E. 2004. V. 70. P. 036602.

[19] Balik S., Kulatunga P., Sukenik C.I., Havey M.D., Kupriyanov D.V., Sokolov I.M. // J. Mod. Opt. 2005. V. 52. P. 2269.

[20] Bienaimé T., Bux S., Lucioni E., Courteille P.W., Piovella N., Kaiser R. // Phys. Rev. Lett. 2010. V. 104. P. 183602.

[21] Sokolov I.M., Kuraptsev A.S., Kupriyanov D.V., Havey M.D., Balik S. // J. Mod. Opt. 2013. V. 60. P. 50.

[22] Chabé J., Rouabah M.T., Bellando L., Bienaimé T., Piovella N., Bachelard R., Kaiser R. // Phys. Rev. A. 2014. V. 89. P. 043833.

[23] Kemp K.J., Roof S.J., Havey M.D., Sokolov I.M., Kupriyanov D.V., Guerin $W$. Optical-depth scaling of light scattering from a dense and cold atomic 87Rb gas. 2018. [Электронный pecypc] Режим доступа: https://arxiv.org/abs/1807.10939.

[24] Roof S., Kemp K., Havey M.D., Sokolov I.M., Kupriyanov D.V. // Opt. Lett. 2015. V. 40. P. 1137.

[25] Skipetrov S.E., Sokolov I.M., Havey M.D. // Phys. Rev. A. 2016. V. 94. P. 013825.

[26] Kuraptsev A.S., Sokolov I.M., Havey M.D. // Phys. Rev. A. 2017. V. 96. P. 023830.

[27] Guerin W., Rouabah M.T., Kaiser R. // J. Mod. Opt. 2017. V. 64. P. 895.

[28] Kuraptsev A.S., Sokolov I.M. // Phys. Rev. A. 2015. V. 91. P. 053822.

[29] Константинов О.В., Перель В.И. // ЖЭТФ. 1960. Т. 39. C. 197.

[30] Келдыш Л.В. // ЖЭТФ. 1964. Т. 47. С. 1515.

[31] Дацюк В.М., Соколов И.М. // ЖЭТФ. 2006. Т. 129. С. 830.

[32] Molisch A., Oehry B. Radiation Trapping in Atomic Vapours. Oxford: Oxford University Press, 1998.

[33] Eloy A., Yao Z., Bachelard R., Guerin W., Fouch M., Kaiser R. // Phys. Rev. A. 2018. V. 97. P. 013810.

[34] Варшалович Д.А., Москалев А.Н., Херсонский В.К. Квантовая теория углового момента. Л.: Наука, 1975.

[35] Соболь И.М. Численные методы Монте-Карло. М.: Наука, 1973.

[36] Datsyuk V.M., Sokolov I.M., Kupriyanov D.V., Havey M.D. // Phys. Rev. A. 2006. V. 74. P. 043812.

[37] Datsyuk V.M., Sokolov I.M., Kupriyanov D.V., Havey M.D. // Phys. Rev. A. 2008. V. 77. P. 033823.

[38] Ларионов Н.В., Соколов И.М. // Квант. электрон. 2007. T. 37. № 12. C. 1130.

[39] Kazakov G.A., Litvinov A.N., Matisov B.G., Romanenko V.I., Yatsenko L.P., Romanenko A.V. // J. Phys. B. 2011. V. 44. N 23. P. 235401.

[40] Barantsev K.A., Velichko E.N., Litvinov A.N. // J. Phys. B. 2014. V. 47. P. 245401. 